\title{
MODELING HUMAN LEARNING INVOLVED IN CAR DRIVING
}

\author{
P.H. WEWERINKE \\ Department of Applied Mathematics \\ University of Twente \\ P.O. Box 217, 7500 AE Enschede \\ The Netherlands
}

\begin{abstract}
In this paper car driving is considered at the level of human tracking and maneuvering in the context of other traffic. A model analysis revealed the most salient features determining driving performance and safety.

Learning car driving is modelled based on a system theoretical approach and based on a neural network approach. The aim of this research is to assess the relative merit of both approaches to describe human learning behavior in car driving specifically and in operating dynamic systems in general.
\end{abstract}

\section{Introduction}

Road traffic performance and safety is determined by several aspects, one of which is the car driving behavior of the human operator. This is the subject of this paper.

Car driving is considered in terms of lane keeping and car following or overtaking slower vehicles, avoiding collisions with oncoming cars. These tasks are analysed and modeled in Chapter 2. The result is a relationship between a variety of task and human operator related parameters and measures of safety and average driving speed (traffic performance).

Several model aspects can be related to the driver's experience level. Learning the driving task is discussed in Chapter 3. Two approaches are followed to model learning. The first one is based on system theory. Learning is modeled as an adaptive estimation process of unknown model parameters. The second approach utilizes a neural network to describe adaptively the input-output behavior of learning the driving task (by adjusting the neural network weights).

Apart from the interest in car driving itself, the study is motivated to compare the two approaches and their relative benefit to describe and predict human learning behavior. For this purpose a simulation program is set up. Preliminary simulation results are presented in Chapter 4

\section{Model analysis of car driving}

The overall goal of car driving is to go from $A$ to $B$ in a certain way (safely, in a given time, etc.). The principal tasks derived from this are lane keeping and overtaking slower vehicles, avoiding a collision with oncoming cars, based on visual cues of the outside world.

\section{$2.1 \quad$ Lane keeping}

Lane keeping is based on two primary visual cue: : the inclination of (or distance to) the road side and tne direction of an aimpoint. The first visual cue prcvides information about the lateral position $y$ (see Figure 1). The direction of an aimpoint $\psi_{a}$ at distance $d$ ahead is given by

$\psi_{a}=\psi+y / d$

Equation (1) shows that for large $d, \psi_{a} \approx \psi$ and for small $d \psi_{a} \approx y / d$; in other words: depending on the 'looking' distance ahead $d$, the driving task resembles more a (relatively easy) heading control task or a (relatively difficult) position control task. This can be illustrated by a simple root locus analysis.

Assuming that the driver is generating a steering wheel deflection $\delta$ proportional to the system output $o$ (i.e., $\psi, y$ or $\psi_{a}$ ), the closed loop system dynamics can be visualized by the poles of the root loci shown in Figure 2 , containing also the coresponding transfer functions $\frac{0}{\delta}(s)$.

The figure shows that good heading control performance can be obtained. Position feedback results in unstable behavior. Thus driver compensation is required (e.g. by means of a heading inner loop). The difference between aimpoint control and position control is the additional lead (zero at $-u / d$, with $u$ the forward driving speed) corresponding to the implicit heading feedback. For large $d$, the zero effectively cancels one of the free poles, and the task approaches the heading control task, yielding system stability, but at the cost of a low frequency path mode (sluggisch response). So

\section{0-7803-2129-4/94 \$3.00 ㄷ 1994 IEEE}


an optimal value of $d$ is determined by the trade-off between stability and performance. In the following the value of $d$ will be related to the driver's experience level.

\subsection{Overtaking}

The decision to pass a slower preceding car is based on the estimated distances to the preceding and oncoming cars. The passing maneuver requires a given distance, $X_{k}$ in the opposite lane, which should be smaller than the available distance between oncoming vehicles. $X_{k}$ depends on the distance $X_{j}$ from the preceding car at the moment of accelerating (determining the driving speed on the opposing lane). This relationship is derived in [1] and given by

$X_{k} \doteq S_{k}+\frac{u_{m}+u_{k}}{u_{m}-u_{j}} \cdot S_{j}+T\left(u_{j}+u_{k}\right) e^{-\sqrt{\frac{2 X_{j}}{T\left(\varepsilon_{m}-u_{j}\right)}}(2)}$

In Figure 3, most of the parameters of Equation (2) are clarified. $S_{k}$ is the minimum distance to car $k, S_{j}$ is the distance which car $i$ is overtaking in the left lane with respect to car $j$, by accelerating from $u_{i_{0}}=u_{j}$ to $u_{m}$ with a first order time constant $T$.

At distance $X_{j}$ from $j$ car $i$ is starting to accelerate determined by the time that the last car before car $k$ has passed. Because of the increased speed it takes a shorter time to overtake car $j$ (to cover the distance $S_{j}$ ). For that reason the resulting required distance between oncoming vehicles (which should be larger than $X_{k}$ ) is decreasing with increasing $X_{j}$. This tradeoff between $X_{k}$ and $X_{j}$ is shown in Figure 4.

Slower cars require (of course) a larger $X_{k}$, especially when also their maximum speed $u_{m}$ is smaller (yielding a larger $\left.X_{k_{\text {min }}}\right)$. The latter is not assumed in the Figure. The Figure also reveals that slower cars can obtain a larger reduction in $X_{k}$ by increasing $X_{j}$.

This possibility to tradeoff $X_{k}$ and $X_{j}$ allows a car to optimize its overtaking strategy depending on the momentaneous traffic situation. This situation can statistically be specified in terms of two probability density functions of the actual distances between the right-lane and left-lane cars, denoted by $p_{a_{j}}$ and $p_{a_{k}}$, respectively. These determine the available spacing between the cars. This is summarized in Figure $5 . X_{k_{0}}$ and $X_{j_{0}}$ represent the minimum car distances and $X_{k_{1}}$ corresponds with $X_{j_{0}}$.

As can be seen in the figure increasing $X_{j}$, yielding a reduction in $X_{k}$, increases the probability that the available distance to the oncoming car $k$ is larger than the required $X_{k}$, and thus will result in superior overtaking performance. Of course, the distance $X_{j}$ should be available, depending on the available car distances in the right lane. Furthermore, the possible $X_{j}$ is contingent on the observable distance to the oncoming car $k$ at the moment of accelerating $\left(X_{k}^{\prime}\right)$. This distance is rapidly increasing with increasing $X_{j}$.

The foregoing issues will be considered in chapter 4 discussing the simulation results.

\section{Learning}

For many practical questions it is important to c perationalize the experience level of the driving task and to have insight in the learning process involved.

In this chapter learning involved in car driving is discussed following two approaches: a system theoretic approach and a neural network approach. The overall objective is to assess the relative benefit of both methods to describe the adaptive characteristics of human control tasks.

\subsection{System theoretic approach}

The system theoretic approach is based on a model of the system and the task but only partly known. Learning is described as an adaptive estimation process of the task based on new data (experience). Furthermc re, it is assumed that for the naive driver the system outputs (visual cues) are partly known. In addition, adaptive control is assumed. For the lane keeping task tais is modeled in terms of varying weightings (tradeoffs) in the performance index which the human operator is assumed to optimize, or directly in terms of feedback control gains. Learning the overtaking maneuver :s described as an adaptive estimation process of unknown parameters.

The partly known system model is given by

$x_{k+1}=A(\theta) x_{k}+B(\theta) u_{k}+E w_{k}$

$y_{k}=C(\theta) x_{k}+v_{k}$

with $x, u, w$ and $y$ the state, control, disturbance and output vector, respectively. It is assumed that uncertainty about the system can be related to unknown parameters $(\theta)$ in the system model. Learning is then modelled as a parameter estimation problem. The procedure to solve this is by adding the unknown pirameters to the state vector (using the parameter model $\theta_{k+1}=\theta_{k}$ ) yielding an augmented nonlinear system

$\bar{x}_{k+1}=f\left(\bar{x}_{k}, u_{k}\right)+\bar{E} w_{k}$

$y_{k}=h\left(\bar{x}_{k}\right)+v_{k}$ 
with

$f=\left[\begin{array}{c}A(\theta) x+B(\theta) u \\ \theta_{k}\end{array}\right] ; \quad \bar{E}=\left[\begin{array}{c}E \\ 0\end{array}\right]$

and

$h=C(\theta) x$

This can be solved by means of an extended Kalman filter to estimate $\bar{x}$ and thus $x$ and $\theta$.

In addition to estimating the partly unknown system, learning can be related to adaptive control behavior.

Lateral car control amounts to feedback control of heading $\psi$ and lateral position $y$. Thus

$\delta=\ell_{1} \psi+\ell_{2} y$

Therefore, learning the optimal control strategy can be related to learning the optimal values of $\ell_{1}$ and $\ell_{2}$. This can be modelled by adjoining the control to the state vector $\bar{x}$ (of equation (3)) and consider $\ell_{1}$ and $\ell_{2}$ as unknown model parameters. These can be treated as the unknown parameters $\theta$ in the system model of equation (3), yielding estimates of $\ell_{1}$ and $\ell_{2}$ by means of the extended Kalman filter.

The optimal overtaking maneuver is based on the functional relationship between $X_{k}$ and $X_{j}$ i.e. $X_{k}=f\left(X_{j}\right)$ as shown in Figure 5. Learning the optimal maneuvering strategy involves the estimation of $f$. This, again can be considered as an estimation problem of unknown model parameters in $f$ as discussed before.

The adaptive estimation process starts with an initial estimate $\hat{X}_{0}$. It is a nontrivial question how the prior knowledge of naive car drivers can be translated into $\hat{X}_{0}$. Experience, in terms of new data $y_{k}$ of equation (4) results in improved knowledge of the system and a better task performance.

\subsection{Neural network approach}

Human operator behavior can be described as the relationship between task inputs $y$ and control outputs $u$ (inputs to the system). Learning this functional relationship between $y$ and $u$ can be described by a neural network (NN).

A NN consists of a number of processing elements with weighted connections. The weights represent the memory of the network and reflect the input-output relationship. The NN can have a given structure (e.g. feedforward) and a given learning strategy (e.g. backpropagation) as discussed in [3].
Human operator learning is described in terms of adjusted weights of the $\mathrm{NN}$ based on input-output data of real life tasks. The NN assumes no specific structure of the input-output relationship but requires data to be trained. Only when these are available a NN model can be 'built' and used for further analysis of learning, etc.

For the car driving task (see References [1] and [4]) the human operator inputs $y$ consist, for the lateral task, of heading and lateral deviation and, for the overtaking task, of speed and relative distances (to preceding and oncoming cars). The outputs $u$ consist of steering wheel deflection, gas and brakes. In Reference [4] preliminary results are discussed to train a $\mathrm{NN}$ for the aforementioned car driving tasks.

\section{Simulation results}

As a first step the lane keeping task was considered. A linear system model was assumed [1] to relate heading and lateral position to the steering angle, with a driving speed of $30 \mathrm{~m} / \mathrm{s}$. Human control response was modelled as a first order lag with a time constant of 0.5 second and an optimal gain.

The task considered was a commanded lane change of 1 meter. After 10 seconds an additional change of $i$ meter was commanded. Ten seconds later a reduction of the lateral position to, again, 1 meter was commi nded and, finally, after 10 seconds a reduction to zero was commanded.

The system model response is shown in Fig. 6. The model exhibits a stable response but with a delay of about 5 seconds (mainly determined by the aimpoint distance).

The NN was trained at the lane keeping task by presenting the system model results of 1000 lane changes between (randomly) 0 and 2 meter at intervals between 0 and 10 seconds. After that, the same commanded lane changes as discussed above were presented to the NN. The resulted response is also given in Fig. 6, showing a rather close agreement with the system model results.

The overtaking task including the system model is described in Chapter 2 was simulated. It was assumed that all cars have a constant speed of $20 \mathrm{~m} / \mathrm{s}$ and that own car $i$ tries to drive $\left(u_{m}\right) 30 \mathrm{~m} / \mathrm{s}$. The actual average speed, in the following expressed as the velocity $v_{r}$ above the $20 \mathrm{~m} / \mathrm{s}$, is the key overtaking performance measure. The experimental (independent) variables considered are the traffic densities in the right and left 
lane $\left(d_{r}\right.$ and $d_{\ell}$, in average number of cars per meter with a Poisson distribution), type of car, in terms of the time constant $\tau$ and overtaking strategy, in terms of the parameter $X_{j}$ (distance to the preceding car at the moment of accelerating).

Figure 7 shows the overtaking performance for two types of cars ( $\tau$ of 5 and 10 seconds) and a given traffic density as a function of the overtaking strategy $\left(X_{j}\right)$. As might be expected from Fig. 5 a relatively small $X_{j}$ improves the overtaking performance substantially. However, beyond 20 to $30 \mathrm{~m}$ the effect becomes small. In Table 1 the overtaking performance is summarized as a function of traffic density, for a given car and overtaking strategy. The density in the right lane $\left(d_{r}\right)$ and in the left lane $\left(d_{\ell}\right)$ are varied independently. Densities of 0.005 result in a performance of $5.2 \mathrm{~m} / \mathrm{s}(18$ $\mathrm{km} / \mathrm{h}$ ) and 181 overtakings (in a simulation period of 2 hours). Especially an increase in the left lane traffic density reduces the performance quickly; for $d_{\ell}=0.02$ hardly any overtakings are still possible.

The next step is to train the NN model based on the system model results and to see how the NN performance will compare with the previous results.

The second step is to simulate the car driving tasks in an experiment with real human operators, with key variable the level of experience.

\section{Concluding remarks}

The model analysis of car driving in Chapter 2 revealed the most interesting aspects of both the lane keeping task and the overtaking task. Several characteristics could be related to the driver's experience level.

Learning the driver's task is discussed and modelled in system theoretical terms. Basically, learning is modelled as an adaptive estimation proces of unknown system- and task parameters. A neural network is considered to model human learning of car driving by describing adaptively the input-output relationship. For this purpose input-output date must be available to train the NN by adjusting the NN parameters.

The aim of this research is to assess the relative merit of both approaches to describe human learning behavior in car driving specifically and in operating dynamic systems in general.

In addition, the human control model will be useful to assess the effect of learning and a variety of task variables, such as traffic density, visibility and road conditions on traffic capacity and safety. Also, a decision support system can be designed, based on the model structure, to facilitate the decision whether a safe overtaking maneuver can be made or not. For this purpose, model predictions can be used to advise more or less experienced drivers with head-up (on the windslield) displays.

\section{References}

[1] P.H. Wewerinke, "Modelling and analysis \& $f$ car driving", Memorandum, Dept. of Applied Mathematics, University of Twente, to appear.

[2] A. Bagchi, Optimal Control of Stochastic Systems, Prentice Hall, 1993.

[3] P.K. Simpson, “Artificial neural systems”, Pergamon Press, 1990.

[4] K.-F. Kraiss and H. Küttelwesch, "Identification and application of neural operator models in a car driving situation", Proc. 5th IFAC Conference on Man-Machine Systems, The Hague, 1992.

\begin{tabular}{|c|c|c|c|}
\hline$d_{\ell}$ & .005 & .01 & .02 \\
\hline \multirow{2}{*}{.005 } & 5.2 & $\begin{array}{l}3.8 \\
\end{array}$ & 2.9 \\
\hline & (181) & (222) & (282) \\
\hline \multirow{2}{*}{.01} & 3.0 & 1.7 & 1.1 \\
\hline & (101) & (98) & (105) \\
\hline \multirow{2}{*}{.02} & 0.3 & 0.2 & 0.1 \\
\hline & (12) & (14) & (11) \\
\hline
\end{tabular}

$\tau=10 s, X_{j}=10 \mathrm{~m}$

(.) number of overtakings

Table 1: Overtaking performance $\left(v_{r}\right)$ as a function of traffic density. 


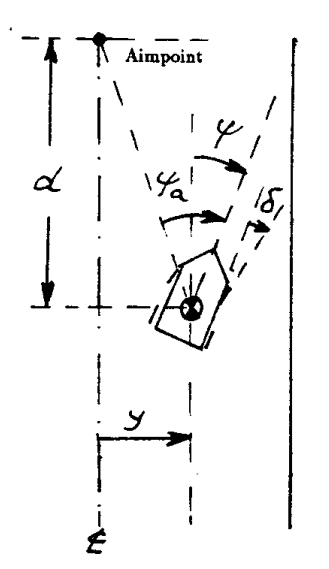

Fig. 1. Lateral driving task situation

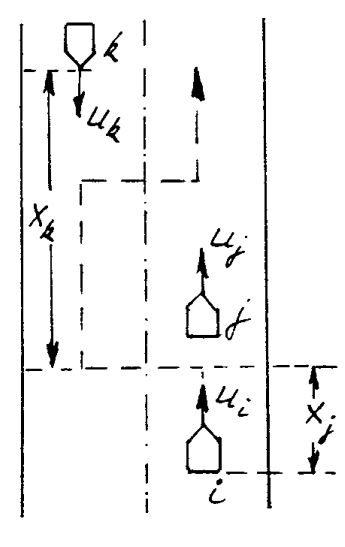

Fig. 3. Driving situation

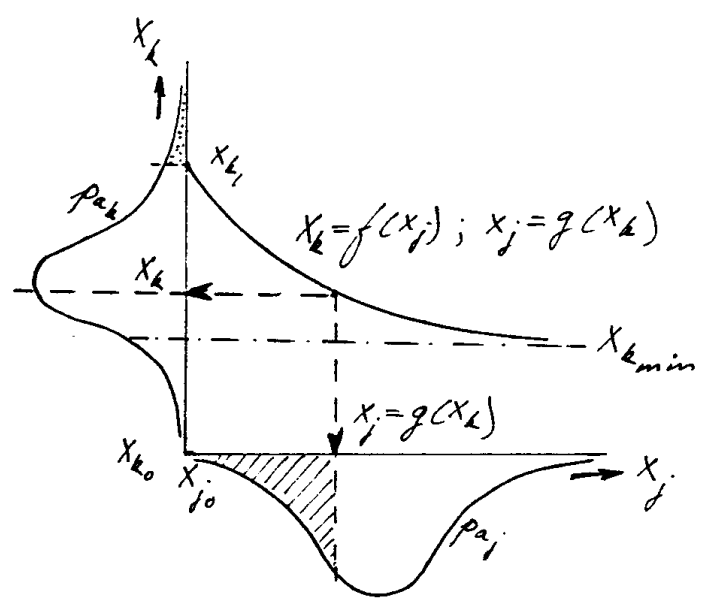

Fig. 5. The statistical relationship between $X$, and $X_{k}$.

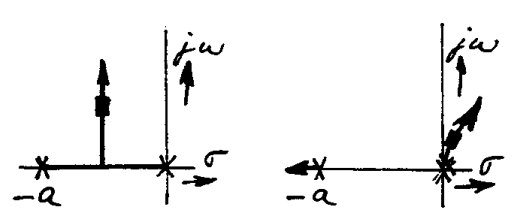

$\frac{y}{\delta}(s)=\frac{K U}{s^{2}(s+a)}$

$\frac{w}{\delta}(s)=\frac{X}{3(s+a)}$

a) heading control

b) position control

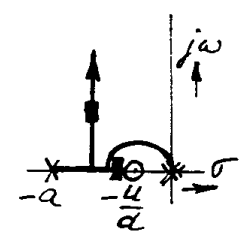

$\frac{w \cdot a}{\delta}(s)=\frac{K(s+u / d)}{s^{2}(s+a)}$

c) aimpoint control

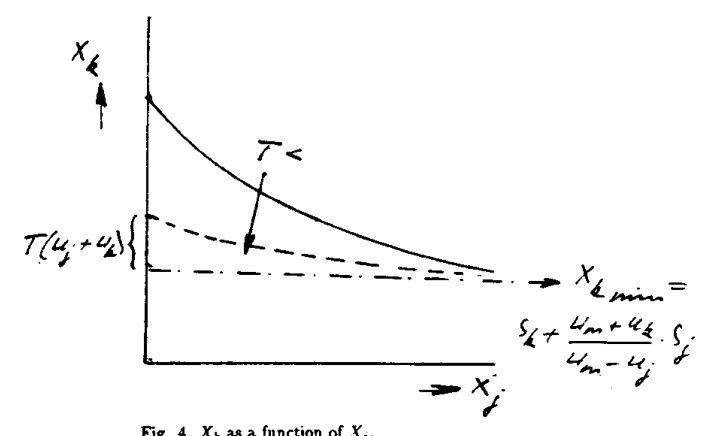

Fig. 4. $X_{k}$ as a function of $X_{j}$.

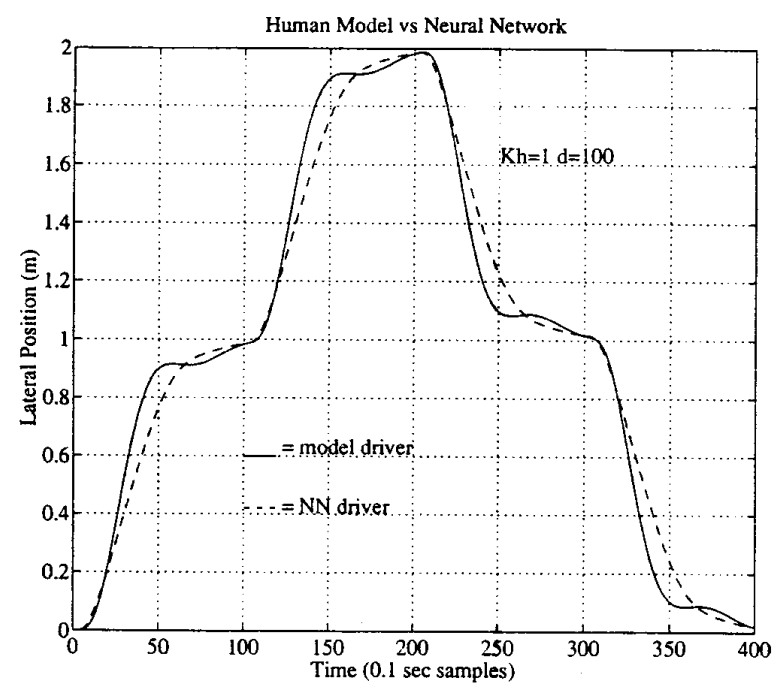

Fig. 6: Lane keeping performance

for the two models. 


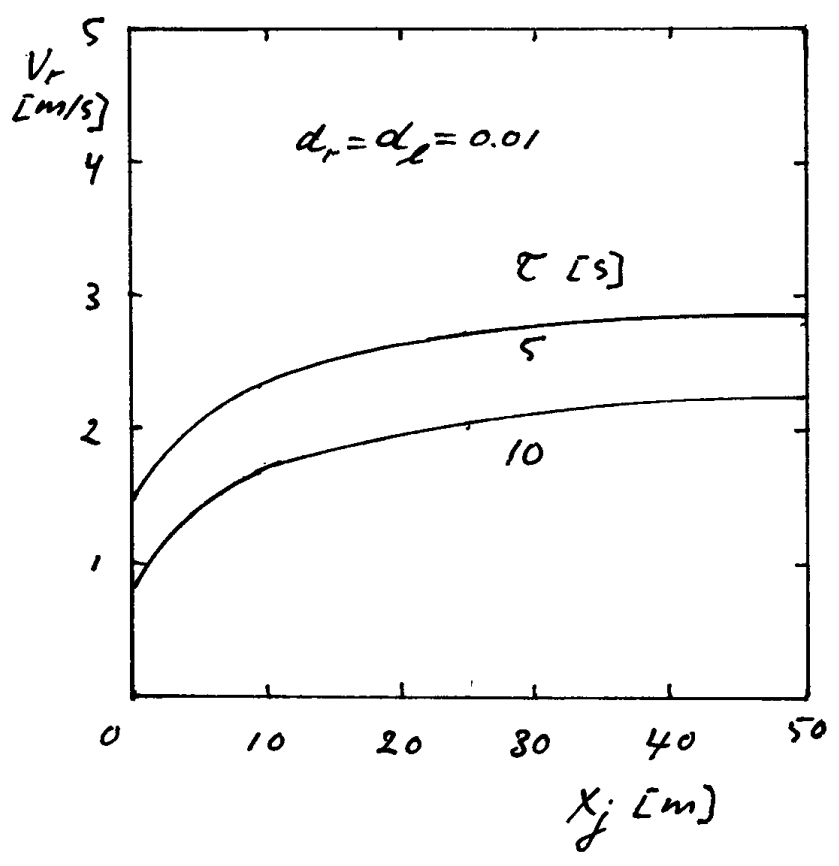

Fig. 7 : Overtaking performance as a

function of overtaking strategy. 